preprint UAB-FT-318

hep-lat/9310025

\title{
Ising Model Universality for Two-Dimensional Lattices*
}

\author{
Wolfhard Janke ${ }^{1}$, Mohammad Katoot ${ }^{2}$ and Ramon Villanova ${ }^{2,3}$ \\ 1 Institut für Physik, Johannes Gutenberg-Universität Mainz \\ 55099 Mainz, Germany \\ 2 Department of Physical Sciences, Embry-Riddle Aeronautical University \\ Daytona Beach, Florida 32114, USA \\ ${ }^{3}$ Grup de Física Teòrica and IFAE, Facultat de Ciències, Universitat Autònoma de Barcelona \\ 08193 Bellaterra, Spain
}

\begin{abstract}
We use the single-cluster Monte Carlo update algorithm to simulate the Ising model on two-dimensional Poissonian random lattices of Delaunay type with up to 80000 sites. By applying reweighting techniques and finite-size scaling analyses to time-series data near criticality, we obtain unambiguous support that the critical exponents for the random lattice agree with the exactly known exponents for regular lattices, i.e., that (lattice) universality holds for the two-dimensional Ising model.
\end{abstract}

\footnotetext{
${ }^{*}$ Work supported in part by The Florida High Technology and Industry Council under Contract FHTIC-15423ERAU.
} 


\section{Introduction}

In numerical simulations of many physical systems random lattices [1, 2] are a useful tool to discretize space without introducing any kind of anisotropy. Recent applications of various types of random lattices can be found in a great variety of fields, such as quantum field theory or quantum gravity [2, 3, 国, the statistical mechanics of membranes [5], diffusion limited aggregation [6], or growth models of sandpiles [7], to mention a few. As a consequence of the preserved rotational (or more generally, Poincaré) invariance, spin systems or field theories defined on random lattices are expected to reach the infinite volume or continuum limit faster than on regular lattices. Implicit in this approach is the assumption of (lattice) universality which states that systems defined on lattices of different type should exhibit the same qualitative behaviour once the physical length scale is much larger than the average lattice spacing. While this assumption is known to be true for spin systems on different regular lattices, previous numerical work [8, 9] on random lattices could only give weak evidence that universality holds in this case as well.

In this note we reconsider the Ising model defined on two-dimensional Poissonian円 random lattices constructed according to the Voronoi/Delaunay prescription [2, 4]. In previous work on this model, Espriu et al. [9] have used standard Metropolis Monte Carlo (MC) simulations on lattices with $N=10000$ sites to study the approach of criticality in the low- and hightemperature phase. Here we report high-statistics simulations in the very vicinity of the phase transition, using considerably larger lattices of size up to $N=80000$. To achieve the desired accuracy of the data we made extensively use of recently developed greatly refined MC simulation techniques, such as cluster update algorithms [11, 12] and reweighting methods [13]. As a result of finite-size scaling (FSS) analyses of our data we obtain very strong support for (lattice) universality in this model.

\section{Model}

As partition function we take

$$
Z=\sum_{\left\{s_{i}\right\}} e^{-K E} ; \quad E=-\sum_{\langle i j\rangle} s_{i} s_{j} ; \quad s_{i}= \pm 1,
$$

\footnotetext{
${ }^{1}$ For alternative site distributions see, e.g., Refs. [6, 10].
} 
where $K=J / k_{B} T>0$ is the inverse temperature in natural units and $\langle i j\rangle$ denote nearest-neighbour links of the Delaunay random lattices, computed according to the (dual) Voronoi cell construction as described, e.g., in Ref.[四]. Following Ref. [9] we thus take the relative weights of the links to be constant. The lattice sizes studied are $N=5000,10000,20000,40000$, and 80000 , with three replicas for each of the two smallest lattices, and two replicas for $N=20000$. We always employed periodic boundary conditions, i.e., the topology of a torus. In this case Euler's theorem implies $\bar{q}=6$, where $\bar{q}$ is the lattice average of the local coordination numbers $q$ that vary for Poissonian random lattices between 3 and $\infty$. All our lattices satisfy this rigorous constraint, and also the distributions $P(q)$ agree well with numerical evaluations of exact integral expressions [14]. The highest coordination number we actually observed in our simulations was $q=13$ in the $N=80000$ lattice.

To update the spins $s_{i}$ we employed the single-cluster update algorithm [12] which is straightforward to adopt to random lattices. From comparative studies 15 on regular lattices the single-cluster update is expected to be more efficient than the multiple cluster variant [11]. All runs were performed at $K=0.263$, the estimate of the critical coupling $K_{c}$ as quoted by Espriu et al. 9]. After discarding from 50000 to 150000 clusters to reach equilibrium from an initially completely disordered state, we generated a further $4 \times 10^{6}$ clusters and recorded every 10th cluster measurements of the energy per spin, $e=E / N$, and the magnetization per spin, $m=\sum_{i} s_{i} / N$ in a timeseries file. From analyses of the autocorrelation functions of $e$ and $m^{2}$ we obtained at the scale of our measurements integrated autocorrelation times of $\hat{\tau}_{e} \approx 0.8-1.3$ and $\hat{\tau}_{m^{2}} \approx 0.7-0.9$, respectively. Our samples thus consist effectively of about 200000 statistically independent data. The statistical errors are estimated by deviding the time series into 20 blocks, which are jack-knived to avoid bias problems in reweighted data.

\section{Results}

To determine the transition point $K_{c}$ and the correlation length exponent $\nu$ we first concentrated on the magnetic Binder parameter [16],

$$
U_{L}(K)=1-\frac{\left\langle m^{4}\right\rangle}{3\left\langle m^{2}\right\rangle^{2}},
$$


where $L \equiv \sqrt{N}$ is defined as the linear length of the lattice in natural units. It is well known [16] that the curves $U_{L}(K)$ for lattices of size $L$ and $L^{\prime}$ should intersect in points $\left(K^{\times}\left(L, L^{\prime}\right), U^{\times}\left(L, L^{\prime}\right)\right)$ which approach $\left(K_{c}, U^{*}\right)$ for large $L, L^{\prime}$, and the derivatives $U_{L}^{\prime} \equiv d U_{L} / d K$ at these points should scale asymptotically with $L^{1 / \nu}$. Our results for $U_{L}(K)$ obtained from reweighting the time-series data at $K=0.263$ are plotted in Fig. 1. For the small lattices the curves are an average over the different replicas [17].

Taking as estimate for $K_{c}$ the average of the $K^{\times}\left(L, L^{\prime}\right)$ for the three largest lattices, we obtain

$$
K_{c}=0.2630 \pm 0.0002,
$$

where the (rough) error estimate reflects also the fluctuations between different replicas. The value (3) is in very good agreement with high-temperature series expansion analyses $\left(K_{c} \approx 0.26303\right)$ [9] and MC simulations in the disordered phase $\left(K_{c}=0.2631(3)\right)$. 9 .

At the critical coupling (3), $U_{L}(K)$ varies only little and an average over all lattice sizes gives

$$
U^{*}=0.6123 \pm 0.0025 .
$$

At $K=K_{c}-0.0002$ and $K=K_{c}+0.0002$ we obtain $U^{*}=0.6054(25)$ and $U^{*}=0.6183(28)$, respectively. The value (团) is in very good agreement with MC estimates for the regular simple square $(s q)$ lattice which are $U^{*}=$ $0.615(10)$ [18 and $U^{*}=0.611(1)$ [19]. This agreement may be taken as a first indication of lattice universality.

To get an estimate for the exponent $\nu$ we have computed the effective exponents

$$
\nu_{\mathrm{eff}}=\frac{\ln \left(L^{\prime} / L\right)}{\ln \left(U_{L^{\prime}}^{\prime}\left(K^{\times}\right) / U_{L}^{\prime}\left(K^{\times}\right)\right)}
$$

for all possible combinations of $L$ and $L^{\prime}$. Since we do not observe any definite trend of $\nu_{\text {eff }}$ as a function of $L$ and $L^{\prime}$, we quote as our final result for $\nu$ the average over all combinations,

$$
\nu=1.008 \pm 0.022
$$

where the error estimate is the standard deviation of the $\nu_{\text {eff }}$. If we consider only the crossing points with the $N=80000$ curve, the estimate for $\nu$ even sharpens to $\nu=1.0043 \pm 0.0036$. We can thus conclude that our estimate 
of the exponent $\nu$ for the random lattice is fully consistent with the exact regular lattice value of $\nu=1$.

The ratio of exponents $\gamma / \nu$ follows from the scaling of the maxima, $\chi_{\max }^{\prime}(L) \propto L^{\gamma / \nu}$, of the (finite lattice) susceptibility

$$
\chi^{\prime}(K)=K N\left(\left\langle m^{2}\right\rangle-\langle|m|\rangle^{2}\right) .
$$

The curves of $\chi^{\prime}(K)$ obtained by reweighting the primary data at $K=0.263$ are shown in Fig. 2. It is then straightforward to determine the maxima $\chi_{\max }^{\prime}$ for each lattice size $L$, and a straight line fit through all data points in a log-log plot of $\chi_{\max }^{\prime}$ vs $L$ gives

$$
\gamma / \nu=1.7503 \pm 0.0059
$$

with a goodness-of-fit parameter [20] of $Q=0.035$. This is again in perfect agreement with the exact value for regular lattices, $\gamma / \nu=1.75$. We can thus conclude that universality also holds as far as the exponent ratio $\gamma / \nu$ is concerned.

The locations of the susceptibility maxima, $K_{\max }^{\chi^{\prime}}$, should scale for large $L$ according to $K_{\max }^{\chi^{\prime}}=K_{c}+a L^{-1 / \nu}$, where $a$ is a non-universal constant. Assuming $\nu=1$ and performing a linear fit through the $K_{\max }^{\chi^{\prime}}$ of the three largest lattices we obtain $K_{c}=0.262947(77)$ with $Q=0.24$, in good agreement with our earlier estimate from the intersection points of the parameter $U_{L}$.

Having estimated $\nu$ and $\gamma$, all other exponents can in principle be calculated by scaling or hyperscaling relations, e.g., $2 \beta / \nu=d-\gamma / \nu$, where $d$ is the dimension. To get an independent estimate for the exponent ratio $\beta / \nu$ we have considered the FSS behaviour of the magnetization $\langle|m|\rangle$ at its point of inflection, which is given by $\left.\langle|m|\rangle\right|_{\text {inf }}(L) \propto L^{-\beta / \nu}$. From a linear fit through all data points we obtain

$$
\beta / \nu=0.1208 \pm 0.0092,
$$

with $Q=0.10$. Also this result is perfectly compatible with the exact value for regular lattices, $\beta / \nu=0.125$, thus supporting the hyperscaling hypothesis for random lattices as well.

Furthermore, from the asymptotic scaling of the points of inflection, $K_{\text {inf }}^{\langle|m|\rangle}=K_{c}+a^{\prime} L^{-1 / \nu}$, we can get another estimate for the critical coupling. Assuming again $\nu=1$, a fit through the points of the three largest 
lattices yields $K_{c}=0.26304(14)$ with $Q=0.60$, thus confirming our previous estimates.

Let us finally consider the specific heat,

$$
C=K^{2} N\left(\left\langle e^{2}\right\rangle-\langle e\rangle^{2}\right),
$$

and the associated critical exponent $\alpha$. Here hyperscaling predicts $\alpha=2-d \nu$. Since we already know that $\nu \approx 1$ we thus expect $\alpha \approx 0$ for two-dimensional random lattices. The corresponding FSS prediction for the maxima of $C$ is then

$$
C_{\text {max }}(L)=B_{0}+B_{1} \ln L,
$$

with non-universal constants $B_{0}$ and $B_{1}$. The semi-log plot in Fig. 3 clearly demonstrates that our data is consistent with this prediction. A linear fit through all data points gives $B_{0}=0.346(52)$ and $B_{1}=0.391(12)$ with $Q=0.84$. On the other hand, we cannot claim unambiguous support for logarithmic scaling. In fact, we can even fit the data with a pure power-law Ansatz, $C_{\max } \propto L^{\alpha / \nu}$, yielding $\alpha / \nu=0.1824(53)$ with a similar goodness-offit parameter, $Q=0.93$, as for the logarithmic fit. We also tried a non-linear three-parameter fit to the more reasonable Ansatz $C_{\max }=b_{0}+b_{1} L^{\alpha / \nu}$. Even though the exponent ratio $\alpha / \nu=0.17(16)$ then comes out consistent with zero, the errors on all three parameters are much too large to draw a firm conclusion from such a fit. By means of exact results for the $s q$ lattice [21], we have checked [17] that for the regular lattice the specific heat behaves very similar. In both cases one would need much larger lattice sizes to discriminate between logarithmic and power-law scaling.

As before the peak locations $K_{\max }^{C}$ should scale like $K_{\max }^{C}=K_{c}+a^{\prime \prime} L^{-1 / \nu}$. Assuming again $\nu=1$, we obtain from a fit to the data for the three largest lattices $K_{c}=0.26295(33)$ with $Q=0.95$, in agreement with the previous estimates.

\section{Conclusion}

In summary, we have performed a fairly detailed finite-size scaling study of the Ising model on two-dimensional Poissonian random lattices of the Delaunay type. Our estimate for the critical coupling derived from the intersection points of the Binder parameter is $K_{c}=0.2630(2)$, the inflection points of the magnetization yield asymptotically $K_{c}=0.26304(14)$, and 
from the peak locations of the suceptibility and specific heat we extrapolate $K_{c}=0.262947(76)$ and $K_{c}=0.26295(33)$, respectively. These values are in good agreement with previous simulations in the disordered phase and with analyses of high-temperature series expansions by Espriu et al. [9].

As usual the specific-heat maxima are difficult to analyze, since the asymptotic finite-size scaling behaviour sets in only for extremely large lattice sizes. Our data is consistent with a logarithmic scaling, i.e., with a critical exponent $\alpha=0$, but not yet sufficient to exclude a power-law scaling with $\alpha \neq 0$ on a statistically firm basis. Precisely the same situation is encountered, however, for the (exactly known) specific heat of the regular $s q$ lattice. We take this observation as further support that also for this quantity there is no violation of universality.

Our results for the critical exponents $\nu, \gamma$ and $\beta$ are much easier to interpret. They clearly indicate that these exponents have the same values as for regular lattices, i.e., here we obtain strong support for lattice universality in the two-dimensional Ising model.

As a future project it would be interesting to repeat this study for $d y$ namical random lattices that satisfy the Voronoi/Delaunay construction at all times [22]. The important question would be whether the critical behaviour is still governed by the critical exponents of the static random (or regular) lattice considered here, or by the critical exponents predicted by matrix model theory [23]. For standard dynamically triangulated lattices, which do not satisfy the Voronoi/Delaunay construction, strong numerical evidence for the second alternative was reported recently in Ref. 224].

\section{Acknowledgements}

R.V. is supported by a fellowship from the "Centre de Supercomputació de Catalunya", and W.J. thanks the Deutsche Forschungsgemeinschaft for a Heisenberg fellowship. Some of the simulations were performed on the SCRI cluster of fast RISC workstations. 


\section{References}

[1] J.L. Meijering, Philips Res. Rep. 8, 270 (1953); R. Collins, in Phase Transitions and Critical Phenomena, Vol. 2, ed. C. Domb and M.S. Green (Academic Press, London, 1972), p. 271; J.M. Ziman, Models of Disorder (Cambridge University Press, Cambridge, 1976); C. Itzykson and J.-M. Drouffe, Statistical Field Theory (Cambridge University Press, Cambridge, 1989), Vol. 2.

[2] N.H. Christ, R. Friedberg, T.D. Lee, Nucl. Phys. B202, 89 (1982); Nucl. Phys. B210 [FS6], 310, 337 (1982).

[3] C. Itzykson, Fields on Random Lattices, in Progress in Gauge Field Theories, proceed. Cargèse Summer School, ed. G.'t Hooft (Plenum Press, New York, 1983).

[4] R. Friedberg and H.-C. Ren, Nucl. Phys. B235[FS11], 310 (1984); H.-C. Ren, Nucl. Phys. B235[FS11], 321 (1984).

[5] F. David and J.-M. Drouffe, Nucl. Phys. B (Proc. Suppl.) 4, 83 (1988).

[6] C. Moukarzel and H.J. Herrmann, J. Stat. Phys. 68, 911 (1992).

[7] H. Puhl, preprint HLRZ 16/93, Jülich (1993).

[8] H.-C. Hege, Diploma thesis, FU Berlin (1984), unpublished; J.F. McCarthy, Nucl. Phys. B275 [FS17], 421 (1986); W.-Z. Li and J.-B. Zhang, Phys. Lett. B200, 125 (1988); H. Koibuchi, Z. Phys. C39, 443 (1988); H. Stüben, Diploma thesis, FU Berlin (1989), unpublished; H. Stüben, H.-C. Hege, and A. Nakamura, Phys. Lett. B244, 473 (1990); J.-B. Zhang and D.-R. Ji, Phys. Lett. A151, 469 (1990).

[9] D. Espriu, M. Gross, P.E.L. Rakow, and J.F. Wheater, Nucl. Phys. B265[FS15], 92 (1986).

[10] G. Le Caër and J.S. Ho, J. Phys. A23, 3279 (1990).

[11] R.H. Swendsen and J.-S. Wang, Phys. Rev. Lett. 58, 86 (1987).

[12] U. Wolff, Phys. Rev. Lett. 62, 361 (1989). 
[13] A.M. Ferrenberg and R.H. Swendsen, Phys. Rev. Lett. 61, 2635 (1988); and Erratum, ibid. 63, 1658 (1989).

[14] J.-M. Drouffe and C. Itzykson, Nucl. Phys. B235[FS11], 45 (1984).

[15] U. Wolff, Phys. Lett. B228, 379 (1989); C.F. Baillie and P.D. Coddington, Phys. Rev. B43, 10617 (1991).

[16] K. Binder, Z. Phys. B43, 119 (1981).

[17] W. Janke, M. Katoot, and R. Villanova, in preparation.

[18] K. Binder and D.P. Landau, Surf. Sci. 151, 409 (1985).

[19] D.W. Heermann and A.N. Burkitt, Physica A162, 210 (1990).

[20] W.H. Press, B.P. Flannery, S.A. Teukolsky, and W.T. Vetterling, Numerical recipes - the art of scientific computing (Cambridge University Press, Cambridge, 1986).

[21] A.E. Ferdinand and M.E. Fisher, Phys. Rev. 185, 832 (1969).

[22] K.B. Lauritsen, C. Moukarzel, and H.J. Herrmann, preprint HLRZ 26/93, Jülich (1993). For technical details, see also K.B. Lauritsen, H. Puhl, and H.-J. Tillemanns, preprint HLRZ 30/93, Jülich (1993).

[23] V.A. Kazakov, Pis'ma Zh. Eksp. Teor. Fiz. 44, 105 (1986) [English translation in JETP Lett. 44, 133 (1986)]; Phys. Lett. A119, 140 (1986); D.V. Boulatov and V.A. Kazakov, Phys. Lett. B186, 379 (1987); Z. Burda and J. Jurkiewicz, Acta Physica Polonica B20, 949 (1989).

[24] J. Jurkiewicz, A. Krzywicki, B. Petersson, and B. Söderberg, Phys. Lett. B213, 511 (1988); R. Ben-Av, J. Kinar, and S. Solomon, Int. J. Mod. Phys. C3, 279 (1992); S.M. Catterall, J.B. Kogut, and R.L. Renken, Phys. Rev. D45, 2957 (1992); C.F. Baillie and D.A. Johnston, Mod. Phys. Lett. A7, 1519 (1992). 


\section{Figure Headings}

Fig. 1: The parameter $U_{L}(K)$ vs the inverse temperature $K$ for random lattices of size $L=\sqrt{N}$ with $N=5000,10000,20000,40000$ and 80000 . The curves are obtained by reweighting the time-series data at $K=0.263\left(\approx K_{c}\right)$.

Fig. 2: The (finite lattice) susceptibility $\chi^{\prime}(K)$ for the same random lattices as in Fig. 1. The curves are obtained by reweighting the time-series data at $K=0.263\left(\approx K_{c}\right)$.

Fig. 3: Finite-size scaling plot of the specific-heat maxima $C_{\max }$ vs $\ln L$, where $L=\sqrt{N}$. The solid straight line shows the least-squares fit $C_{\text {max }}=B_{0}+B_{1} \ln L$, with $B_{0}=0.346(52)$ and $B_{1}=0.391(12)$. 
This figure "fig1-1.png" is available in "png" format from: http://arxiv.org/ps/hep-lat/9310025v1 
This figure "fig1-2.png" is available in "png" format from: http://arxiv.org/ps/hep-lat/9310025v1 